\title{
High Resolution Observations of Herbig-Haro Flows
}

\author{
B. Reipurth ${ }^{1}$, S. Andrews ${ }^{1}$, J. Bally ${ }^{2}$ and T.L. Beck ${ }^{3}$ \\ 1: Institute for Astronomy, University of Hawaii, 2680 Woodlawn Dr, \\ Honolulu, HI 96822, USA \\ 2: Center for Astrophysics and Space Astronomy, University of \\ Colorado, Boulder, CO 80309, USA \\ 3: Gemini, 670 N.A'ohoku Place, Hilo, HI 96720, USA
}

\begin{abstract}
Observational techniques with high angular and high spectral resolution have become available in recent years and have had a major impact on the study of Herbig-Haro outflows from very young stars. We discuss new results obtained using WFPC2, ACS and STIS onboard the Hubble Space Telescope, as well as spectroscopic data obtained at the Keck telescope and with the Integral Field Unit at the Gemini telescope. We also discuss centimeter radio continuum maps from the Very Large Array and their importance in testing scenarios for the formation of giant Herbig-Haro flows.
\end{abstract}

\section{Introduction}

Herbig-Haro flows represent the most visible and the most spectacular of the various outflow phenomena associated with stellar youth. In this brief presentation, we give an overview of some recent results of our group in this area, with emphasis on optical and radio high resolution techniques. For space reasons we must leave out a discussion of infrared observations, but refer to Reipurth \& Bally (2001) for a recent more complete review.

\section{HST Observations}

The WFPC2 imager onboard HST has had a major impact on the study of HH flows. The widths of $\mathrm{HH}$ jets have been well resolved by such images, as has the stratification of the cooling zone behind the larger bow shocks. WFPC2 images have given us information about the detailed structure of jets that has offered substantial support to the internal working surface model of Raga et al. (1990). In this view, the knots in jets are due to shocks occuring when faster moving parcels of gas catch up with slower gas. The knots thus result from variability in the driving sources. It is fortunate that the velocities and distances of $\mathrm{HH}$ jets are such that we can precisely measure their proper motions on WFPC2 images on timescales of only a few years. This is important, because such timescales are generally shorter than the postshock cooling times of $\mathrm{HH}$ knots, and thus the cross-correlation techniques employed to derive the proper 


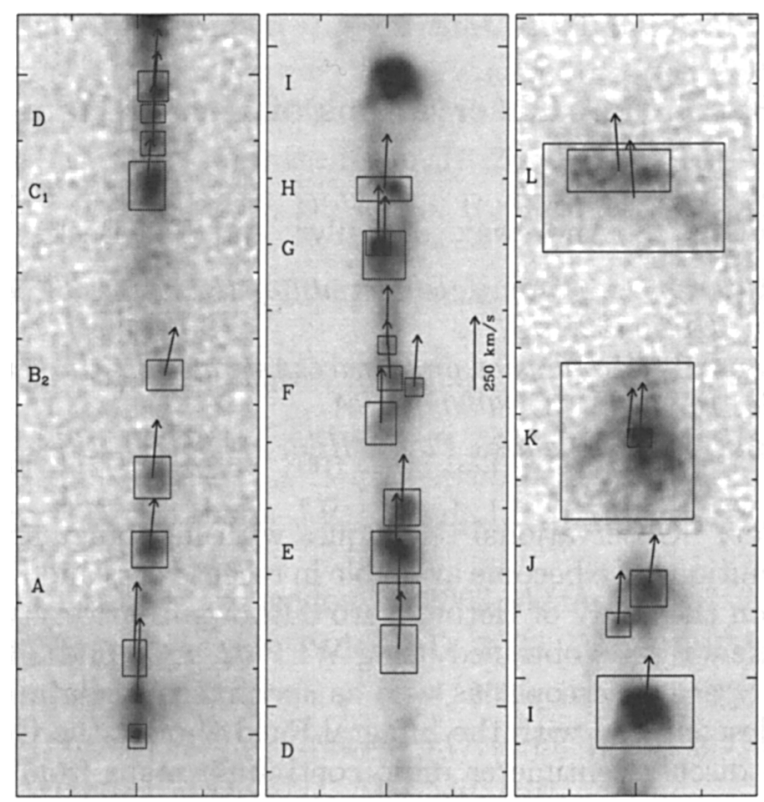

Figure 1. A WFPC2 image in [S II] showing proper motions of the jet knots in $\mathrm{HH} 34$ determined from multi-epoch images.

motions are not seriously affected by variability. Figure 1 shows an example of such proper motions determined for the HH 34 jet (Reipurth et al. 2002).

With the Advanced Camera for Surveys (ACS) on HST, we now have a tool that offers even better resolution $\left(0.05^{\prime \prime} /\right.$ pixel $)$, albeit of the emission lines important for $\mathrm{HH}$ flows only $\mathrm{H} \alpha$ is observable with ACS. Recently, ACS has obtained some stunning images of irradiated jets embedded in the Orion Nebula and the nearby HII region M43. Previous ground-based observations of the Orion region with narrow-band filters and tunable Fabry-Perots led to the discovery of several dozen jets and outflows from young stars in the Orion Nebula (O'Dell et al. 1997; Bally \& Reipurth 2001; Bally et al. 2001). The narrow-band $\mathrm{H} \alpha$ filter on the ACS was used in fall 2002 to obtain high angular resolution images of several fields in the Orion region. One pointing was centered on the bent irradiated jet HH 502 located south of Orion's Bright Bar (Figure 2 top). A second pointing targeted the peculiar one-sided jet $\mathrm{HH} 505$ located in the western part of the Nebula. The ACS observations reveal that the bent HH 502 flow emerges from a bright proplyd. The ionized edge of this object blends smoothly into the southwest-facing red-shifted jet emerging from its central star. Both jet and counter-jet beams are resolved into chains of compact knots and very small bow shocks which terminate in the large bow shocks detected in ground based images. The jets in this system propagate close to the northern rims of the large shocks, indicating that a large-scale outflow from the Orion Nebula is deflecting these flows towards the south. 

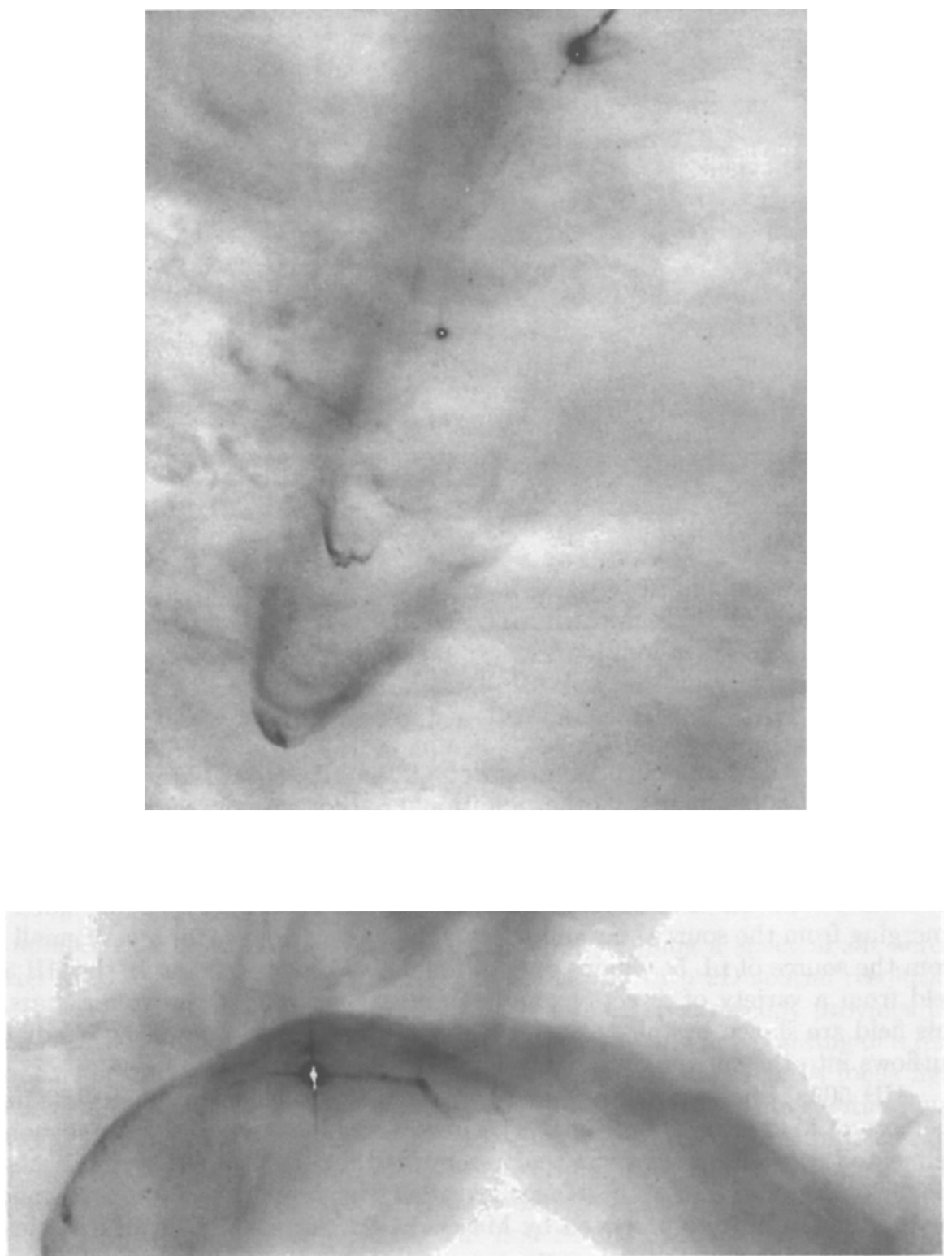

Figure 2. ACS images of HH 502 (top) and HH 505 (bottom) in $\mathrm{H} \alpha$ 


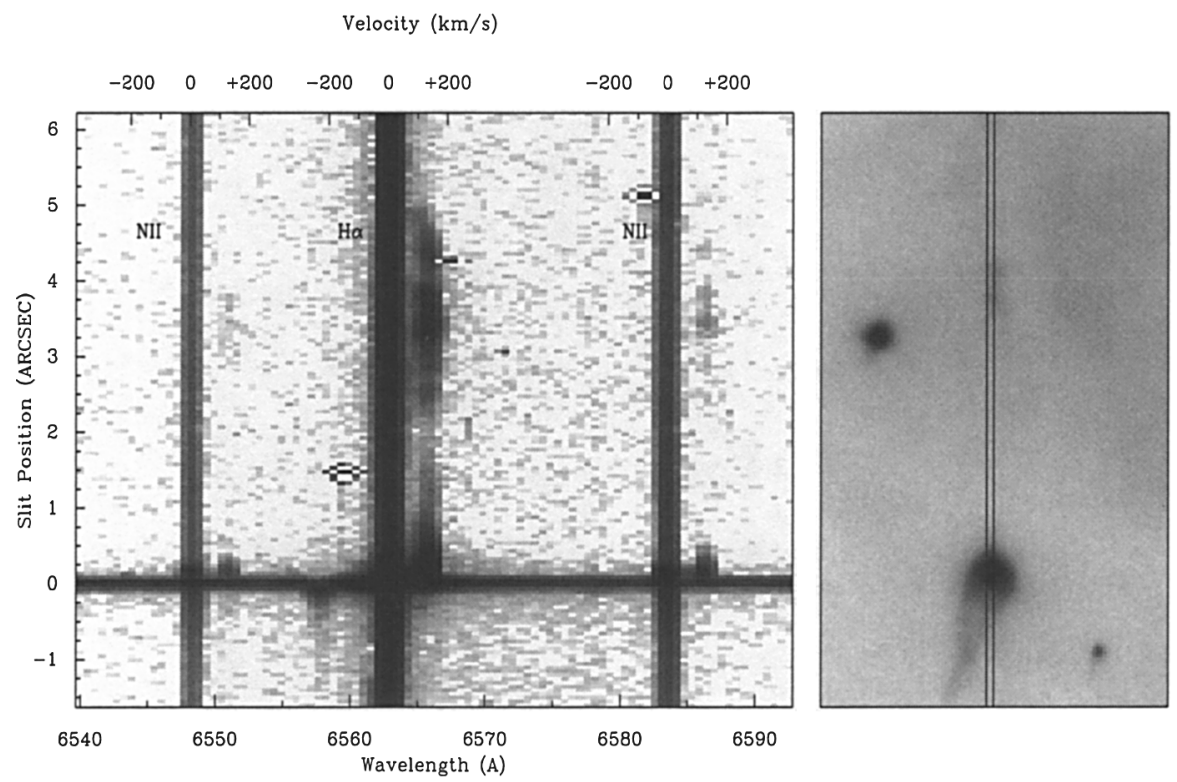

Figure 3. A STIS spectrum of the HH 514 jet emerging from the proplyd 170-337 in M42.

Perhaps the most surprising aspect of the HH 502 ACS observations is that the field contains many other additional outflows, many of which were not apparent in arc-second resolution ground based data. Two parabolic bow shocks surrounding low-mass young stars (the LL Orionis type objects LL 5 and LL 6) are clearly resolved. The object LL 6 is shown to be powered by a one-sided jet emerging from the source star and there is tentative evidence for a very small jet from the source of LL 5. Chains of additional bow shocks criss-cross the HH 502 field from a variety of directions and sources. Over half of the young stars in this field are shown by the ACS observations to be actively powering irradiated outflows into the surrounding photo-ionized nebula.

HH 505 is shown to be powered by a bipolar jet deflected by outflow from the core of M42 (Fig. 2 bottom). The upstream boundary of the jet cocoon is delineated by an $\mathrm{H} \alpha$ bright rim that resembles the other LL Ori type objects in the Orion Nebula. The ACS images provide strong confirmation for the model for the LL Ori objects proposed by Masciadri \& Raga (2001), in which a jet is swept by a side wind.

We have used the Space Telescope Imaging Spectrograph (STIS) on HST to obtain $\mathrm{R}=5000$ spectra of over a dozen proplyds in the Orion Nebula in the vicinity of the $\mathrm{H} \alpha$ line. The advantage of HST for such spectroscopy is that it can provide angular resolution along the slit of better than $0.1^{\prime \prime}$, enabling the analysis of the spatial structure of supersonic jet flows. Figure 3 shows a STIS spectrum of the HH 514 jet emerging from the proplyd 170-337 (HST2) south of the Trapezium stars. This is part of a series of 5 spectra, where each $0.1^{\prime \prime}$ wide slit is displaced from the previous position by $0.01^{\prime \prime}$. 
These observations show kinematic asymmetry; the faint blueshifted jet has a velocity twice as large as the brighter redshifted flow. Interestingly, there is a dip in the stellar $\mathrm{H} \alpha$ profile and continuum that exactly matches the radial velocity and line width of the blueshifted jet. It is possible that this feature is an absorption feature produced by the jet. Such a feature can be produced if the population in the $n=2$ state of recombining hydrogen is sufficiently large. Pumping of this level can occur in the presence of an extremely strong Lyman- $\alpha$ radiation field with a flux of order $10^{14}$ Lyman alpha photons per second and a Lyman- $\alpha$ optical depth of order $10^{7}$. These conditions can be met in a neutral proplyd envelope with a column density $N(H)>10^{21} \mathrm{~cm}^{-2}$ located within $10^{17}$ $\mathrm{cm}$ of the Trapezium stars.

The blueshifted absorption implies that the base region of the jet must completely cover the stellar photosphere from our vantage point. Thus, the jet must start out as a relatively wide-angle wind with an opening angle at least as large as the inclination angle of the jet axis with respect to our line of sight. The optical depth of the absorption feature places a constraint on the column density of this wind along the line of sight and on the distance from the star to the launch point. A preliminary analysis indicates that the absorbing gas must be located within 1 AU of the star.

Though the jet from this proplyd was detected from the ground and seen in the HST images, most proplyds do not show such clear signposts of outflow activity. Yet, most proplyds reveal the presence of highly supersonic features in the STIS spectra. These observations indicate that most proplyds accelerate jets and outflows with mass loss rates larger than $d M / d t=10^{-9} \mathrm{M}_{\odot} \mathrm{yr}^{-1}$. Thus, the disks in most proplyds are active and must be dissipating angular momentum at sufficient rates to sustain jet production.

\section{Integral Field Units}

A number of studies have used the technique of stepping a spectral slit across a Herbig-Haro energy source to obtain spectroscopy with $2 \mathrm{D}$ spatial resolution on the sky (e.g. Bacciotti et al. 2002). Integral field spectroscopy provides the opportunity to obtain such spectral image cubes with only one pointing of a telescope. The Integral Field Units (IFUs) used at optical wavelengths focus light onto a lenslet array where it is redirected to slits, using either fiber optics or an array of slitlets (e.g. Bacon 1995; Allington-Smith et al. 2002). IFU spectroscopy provides data at higher spatial resolution over a smaller field than Fabry-Perot interferometry, and can provide simultaneous coverage of many emission lines of interest in the study of $\mathrm{HH}$ objects. The first results of IFU spectroscopy of an $\mathrm{HH}$ outflow were the spectral images of the DG Tau jet presented by Lavalley et al. (1997).

The IFU in the Gemini Multi-Object Spectrographs (GMOS) at both the Gemini North and Gemini South Observatories provide the opportunity to probe the spatial structure of $\mathrm{HH}$ outflows at spectral resolutions of $\sim 15 \mathrm{~km} / \mathrm{s}$ at $650 \mathrm{~nm}$. The IFU in "one slit mode" results in a field of view of 3. " $5 \times 5^{\prime \prime}$ and simultaneous spectral coverage of $\sim 210 \mathrm{~nm}$, while "two slit mode" has double the field of view with half of the spectral range. The IFU lenslets subtend a $0 .{ }^{\prime \prime} 2$ hexagonal region on the sky, and the tip-tilt and astigmatism correction 
using the GMOS on-instrument wavefront sensor result in seeing-enhanced data. Tasks in the Gemini IRAF package have been constructed to reduce the complex 2D IFU spectra into a 3D spectral image cube format. The GMOS IFU has been used to observe the spectral and spatial structure of HH 32 (Beck et al. 2004a), and regions of the collimated jet associated with the HH 34 outflow (Beck et al. 2004b).

The final 3D spectral data cube of HH 32 covered a $8 . " 7 \times 5 . " 85$ area with $\sim 0 . " 5$ spatial resolution over a 482-704 nm spectral region centered on the $\mathrm{HH}$ 32 knot A complex (Beck et al. 2004a). This spectral coverage allowed line ratio channel maps of [O III] $5007 / \mathrm{H} \alpha$, [O I] $6300 / \mathrm{H} \alpha$, [N II] $6583 / \mathrm{H} \alpha$, [S II] $(6716+6730) / \mathrm{H} \alpha$ and [S II] $6717 / 6731$ to be constructed and analyzed. The radial velocity channel maps of the $\mathrm{H} \alpha$ line observed in $\mathrm{HH} 32 \mathrm{~A}$ are presented in Figure 4, and shows a line of sight superposition of two or three working surfaces located along the redshifted body of the $\mathrm{HH} 32$ outflow. The line emission and line ratios are found to vary significantly on spatial scales of $\sim 1^{\prime \prime}$ and over velocities of $\sim 50 \mathrm{~km} / \mathrm{s}$. A "3/2 dimensional" bow shock model was constructed to describe the observations, and it was qualitatively successful at reproducing the general features of the $\mathrm{H} \alpha$ radial velocity channel maps (Beck et al. 2004a). Based on these data, a variable velocity, precessing jet model for the HH 32 flow has recently been presented by Raga et al. (2004).

Observations with optical IFUs provide an opportunity to study the $3 \mathrm{D}$ electron density structure of outflows from the [S II] 6717/6731 line ratio maps. For the observations of $\mathrm{HH} 32$, the maps of the line ratios show that the peak flux in the $\mathrm{H} \alpha$ emission corresponds to the highest electron density regions, and that these knots of emission are enclosed by a low density envelope (Beck et al. 2004a). In Figure 5, the [S II] line ratio maps of a section of the jet in the collimated HH 34 outflow are presented over the velocity extent of the emission (Beck et al. 2004b). The panel at the top left of the figure shows the scale of the line ratios; values of 0.5 correspond to electron densities of $n_{e} \sim 600 \mathrm{~cm}^{-3}$ and ratios of 1.5 trace regions with $n_{e} \sim 100 \mathrm{~cm}^{-3}$. There is structure observed in the density maps on a sub-arcsecond level. In all velocity slices it appears that the higher electron densities may correspond to the recombination regions at the head of the individual bow shock knots which were mapped in the high spatial resolution HST images of Reipurth et al. (2002). These regions are likely detected as peaks in the electron density maps because of the nearly edge-on orientation of the $\mathrm{HH} 34$ flow.

\section{The Origin of Jets: Long Slit Spectroscopy}

Most jet sources are heavily embedded in their natal molecular material, and the innermost portions of their jets are obscured. The jet launch and collimation regions are thought to be located within an $\mathrm{AU}$ (or much less for the $\mathrm{X}$-wind model) and within a few hundred AU from the outflow source, respectively (e.g. Shu et al. 2000; Königl \& Pudritz 2000). Therefore, to even begin to understand the conditions in these regions in an observational context, one must follow the jet upstream as close to the source as possible. The discoveries of jets resident in $\mathrm{HII}$ regions present an opportunity to attempt direct observations of the launch and collimation regions (Reipurth et al.1998; Cernicharo et al.1998). 
02004006008001000

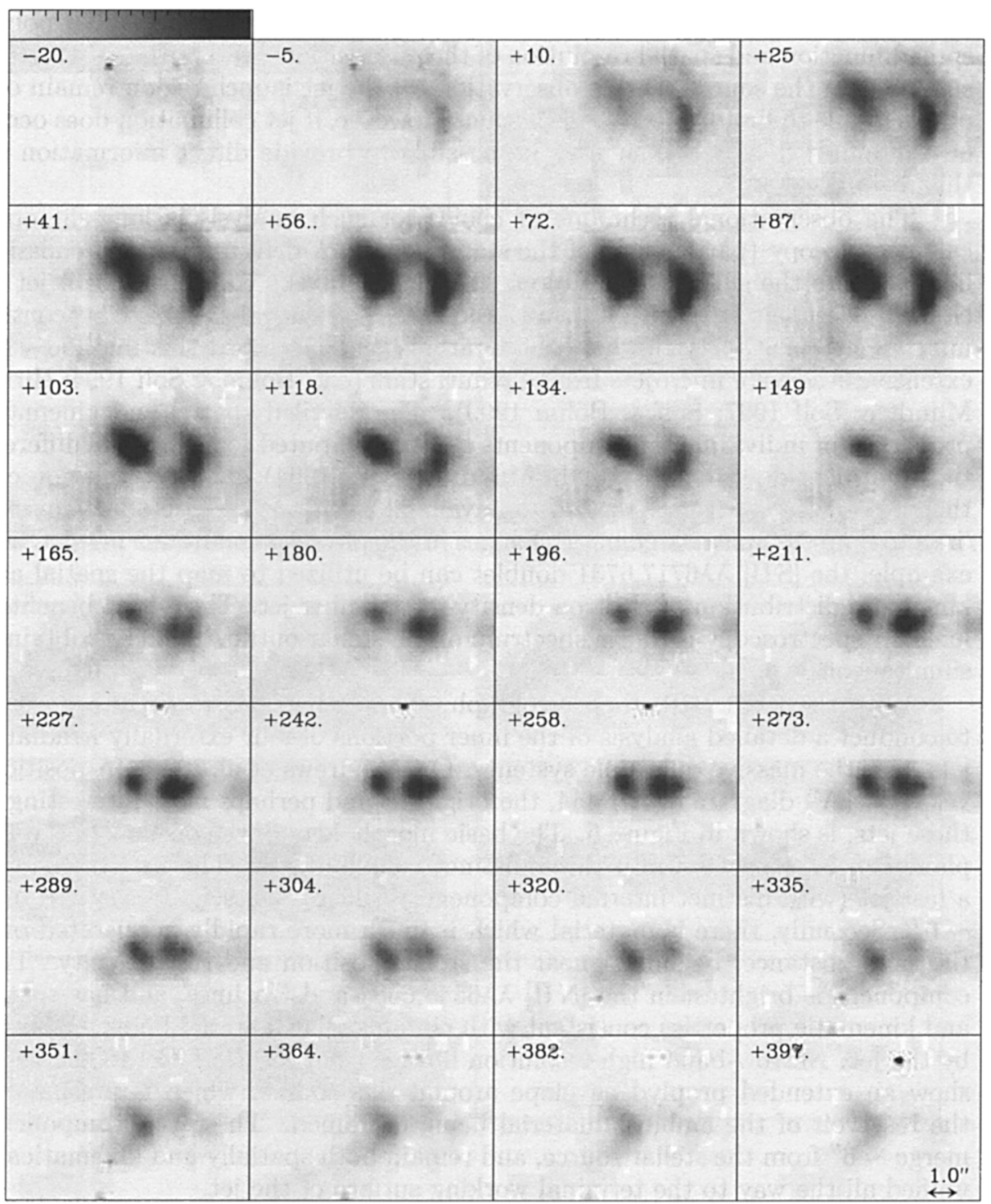

Figure 4. The observed $\mathrm{H} \alpha$ radial velocity channel maps from Gemini IFU observations of HH 32 are plotted with a linear greyscale. The heliocentric radial velocities range from -20 to $+397 \mathrm{~km} \mathrm{~s}^{-1}$. The central heliocentric velocity of each channel is shown in the corresponding panel. The spatial scale is shown in the $+397 \mathrm{~km} \mathrm{~s}^{-1}$ panel. The intensity scale displayed at the top left of the figure is in counts. 
These externally irradiated jets have had their molecular cocoons removed by the UV radiation of nearby massive stars, thereby permitting standard optical jet analysis close to the outflow sources. Therefore, the factors limiting studies of the launch and collimation regions for irradiated jets are primarily the stellar pointspread function and spatial resolution of the observations, rather than extinction surrounding the source. Direct observations of the jet launch region remain out of reach, due to limited spatial resolution. However, if jet collimation does occur on the hundred $\mathrm{AU}$ scale, it may be possible to provide direct information on this region.

The observational technique of choice for such analysis is long-slit optical spectroscopy (particularly of the standard, shock-driven forbidden emission lines), where the slit is aligned along the outflow axis. To investigate a jet as close as possible to the outflow source requires the removal of the stellar continuum. Solf $(1989,1994)$ and his collaborators have developed this method with extensive studies of microjets from T Tauri stars (e.g. Böhm \& Solf 1994; Hirth, Mundt \& Solf 1997; Solf \& Böhm 1999). The detailed spatial and kinematic properties of individual jet components can be computed following the differential treatment described by Hirth, Mundt, \& Solf (1994). This information can then be combined with the diagnostics available from various forbidden emission lines to map the spatio-kinematic changes in the physical conditions in a jet. For example, the [S II] $\lambda \lambda 6717,6731$ doublet can be utilized to map the spatial and kinematic distribution of electron density in the inner jet. The added benefit of long-slit spectroscopy is that a spectrum of the stellar outflow source is obtained simultaneously.

With the Keck HIRES spectrograph, this method has recently been used to conduct a detailed analysis of the inner portions of four externally irradiated jets near the massive quintuple system $\sigma$ Ori (Andrews et al. 2004). A positionvelocity (PV) diagram of $\mathrm{HH} 444$, the brightest and perhaps most interesting of these jets, is shown in Figure 6. The basic morphology of the outflow in the PV plane can be separated into three distinct contributions. The first of these is a fast jet (with distinct internal components) which is slightly decelerated over $\sim 6^{\prime \prime}$. Secondly, there is material which is much more rapidly accelerated over the same distance, beginning near the stellar position and rest velocity. This component is brightest in the [N II] $\lambda \lambda 6548,6584$ and $\mathrm{H} \alpha$ lines, and has spatial and kinematic properties consistent with circumstellar material being entrained by the jet. Narrow-band high-resolution images from the HST WFPC2 camera show an extended proplyd envelope around this source, which is presumably the reservoir of the ambient material being entrained. These two components merge $\sim 6^{\prime \prime}$ from the stellar source, and remain both spatially and kinematically unified all the way to the terminal working surface of the jet.

The physical origin of the third component (labelled E1 in Figure 6) in the PV diagram, a bright, kinematically broad emission knot offset from the stellar position by 0 "' 15 (roughly $70 \mathrm{AU}$ in projected physical separation), is uncertain. Its physical properties lie intermediate to distinct component classes seen in many other jets (e.g., Hirth, Mundt, \& Solf 1997). The relatively small spatial offset from the stellar source indicates a possible connection with the region of collimation. Continued use of long-slit spectroscopy with large telescopes may 


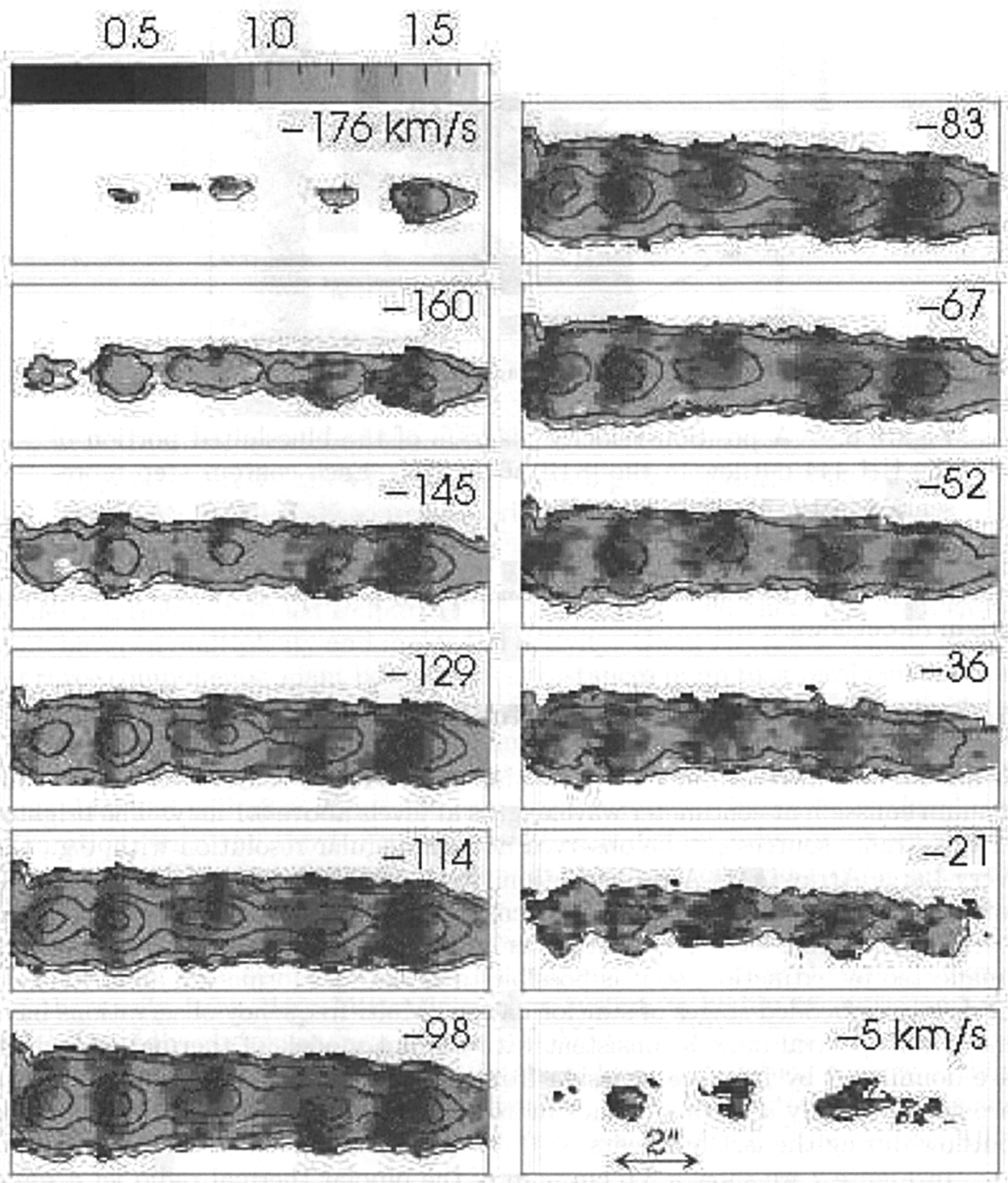

Figure 5. Maps of the [S II] 6717/6731 line ratios in HH 34 that trace electron density are plotted over the velocity extent of the D (right) through J (left) knots of the collimated HH 34 jet. The exciting source is off the field to the right, and the flow direction is to the left. The values of the line ratios are presented in the greyscale at the upper left, and the total emission in the [S II] lines is defined by contours. 


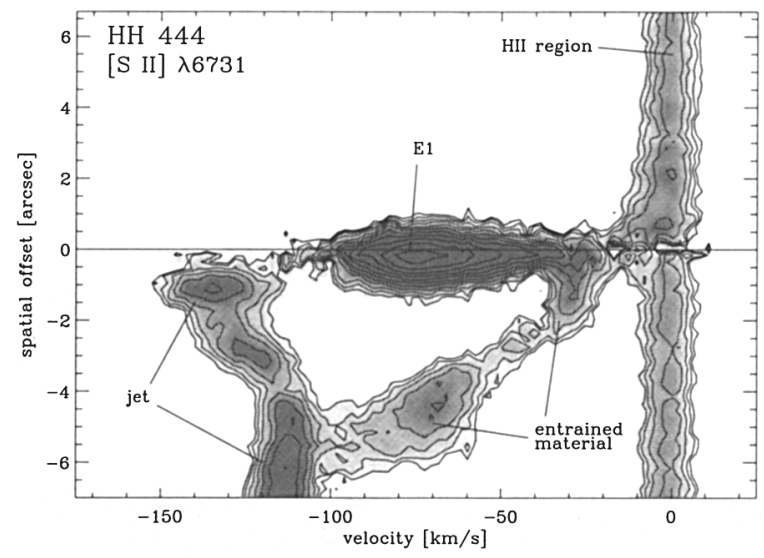

Figure 6. A position-velocity diagram of the blueshifted portion of the HH 444 outflow in the [S II] $\lambda 6731$ line. Each contour step represents a factor of $\sqrt{2}$ in intensity.

reveal more instances of such a component, and perhaps shed new light on the origin of outflows.

\section{VLA Observations and the Multiplicity of Jet Sources}

It has become increasingly clear that almost all outflow sources emit radio continuum emission at centimeter wavelengths at levels above $0.1 \mathrm{mJy}$. The brighter of these radio sources can be observed at high angular resolution with, e.g., the Very Large Array in its A-configuration. Such observations have two major advantages. First, the resolution at $3.6 \mathrm{~cm}$ is very high, about $0.2^{\prime \prime}$. Second, and perhaps most important, at centimeter wavelengths the emission is essentially unaffected by extinction, so it is possible to explore jet formation and evolution at deeply embedded stages of star formation. Multi-frequency observations have measured spectral indices consistent with current models of thermal jets which are dominated by free-free emission from partially ionized gas. The radio jets are generally only a few arcseconds in extent, thus revealing the direction of the outflow during the last few years.

In Figure 7 we show a $3.6 \mathrm{~cm}$ map of the bipolar thermal radio jet around the embedded Class I source IRAS 05329-0505 in Orion, which drives the parsecscale flow HH 41/42 (Reipurth et al. 2004). Examination of the contours reveal a significant wiggling of the flow axis, indicating that the driving source is a binary with a period of about 40 years, suggesting that the semi-major axis is of the order of $10 \mathrm{AU}$.

Even higher resolution observations are possible at a wavelength of $7 \mathrm{~mm}$. This is the shortest wavelengths that can be observed before dust emission significantly impacts the data. Such observations are particularly useful to study the binarity of embedded sources, as demonstrated by, e.g., Loinard et al. (2002).

Various types of observations have revealed a very high multiplicity, around $80 \%$, among the youngest outflow sources, with half of the binaries being higher 


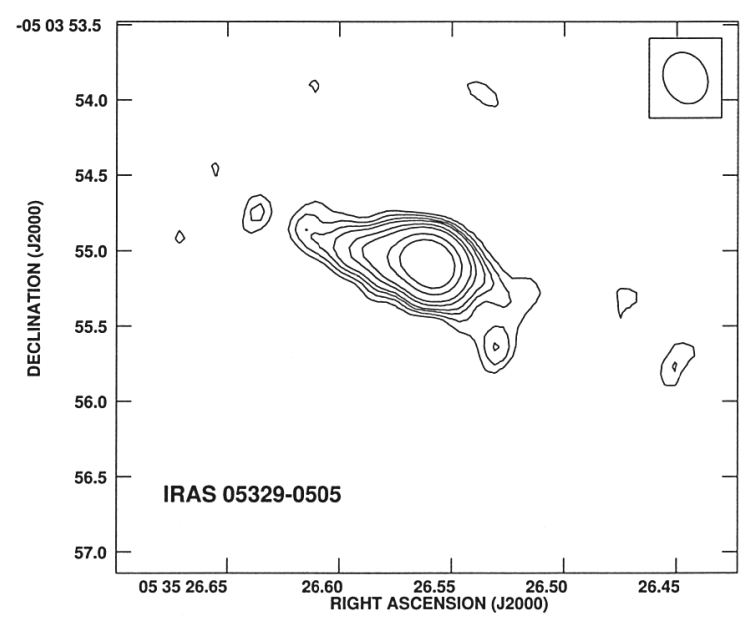

Figure 7. A $3.6 \mathrm{~cm}$ VLA map shows a bipolar thermal radio jet from the IRAS 05329-0505 source in Orion with evidence of precession.

order multiples. This led Reipurth (2000) to suggest that there may be a causal link between multiplicity and major $\mathrm{HH}$ activity. Giant $\mathrm{HH}$ flows broadly consist of three components: giant bow shocks at distances of up to several parsecs from their sources, intermediate knots, and finely collimated jets directly associated with the sources. These categories may find an explanation in the dynamical evolution of multiple systems. Non-hierarchical triple systems are inherently unstable, and will after about 100 crossing-times decay into a hierarchical configuration, with the third member placed in a distant orbit or escaping from the system. This transformation occurs as the result of a close triple approach between the stellar members. Serious disturbances of the circumstellar disks occur during a close triple approach, leading to major outflow activity producing the giant bow shocks. After the formation of a hierarchical triple, the orbit of the newly born close binary in such a system is highly eccentric and will evolve rapidly, shrinking from maybe 100 AU to 10 AU or less. During periastron passages the disks of the stars interact, leading to minor outflow activity on an orbital timescale, resulting in first the knots with intermediate spacing, and finally in the collimated jets, which represents the end of the major outflow phase.

To investigate whether this interpretation of the giant $\mathrm{HH}$ flows is correct, it is clear that an analysis of the multiplicity of deeply embedded very young outflow sources is essential. The new infrared and especially radio techniques are beginning to offer the high angular resolution required to tackle this problem.

\section{References}

Allington-Smith, J. et al. 2002, PASP, 114, 892

Andrews, S. M., Reipurth, B., Bally, J., \& Heathcote, S. 2004, submitted

Bacciotti, F., Ray, T. P., Mundt, R., Eislöffel, J., \& Solf, J. 2002, ApJ, 576, 222 
Bacon, R. 1995, in 3D Optical Spectroscopic Methods in Astrophysics, ASP Conference Series vol. 71, Eds. G. Comte \& M. Marcelin, p.239

Bally, J., \& Reipurth, B. 2001, AJ, 546, 299

Bally, J., Johnstone, D., Joncas, G., Reipurth, B., Mallén-Ornelas, G. 2001, AJ, 122,1508

Beck, T. L., Riera, A., Raga, A.C., \& Aspin, C. 2004a, AJ, in press

Beck, T. L. et al. 2004b, in preparation

Böhm, K.-H., \& Solf, J. 1994, ApJ, 430, 277

Cernicharo, J., Lefloch, B., Cox, P., Cesarsky, D., Esteban, C., Yusef-Zadeh, F., Méndez, D. I., Acosta-Pulido, J., García-López, R. J., \& Heras, A. 1998, Science, 282, 462

Hirth, G. A., Mundt, R., \& Solf, J. 1994, A\&A, 285, 929

Hirth, G. A., Mundt, R., \& Solf, J. 1997, A\&AS, 126, 437

Königl, A., \& Pudritz, R. E. 2000, in Protostars and Planets IV, eds. V. Mannings, A. P. Boss, S. S. Russell (Tucson: Univ. of Arizona Press), p.759

Lavalley, C. et al. 1997, A\&A, 327, 671

Loinard, L., Rodríguez, L.F., D’Alessio, P., Wilner, D., Ho, P.T.P. 2002, ApJ, 581, L109

Masciadri, E., \& Raga, A.C. 2001, AJ, 121, 408

O'Dell, C.R., Hartigan, P., Lane, W.M., Wong, S.K., Burton, M.G., Raymond, J., \& Axon, D.J. 1997, AJ, 114, 730

Raga, A.C., Binette, L., Cantó, J., Calvet, N. 1990, ApJ, 364, 601

Raga, A. et al. 2004, AJ, in press

Reipurth, B. 2000, AJ, 120, 3177

Reipurth, B., \& Bally, J. 2001, ARAA, 39, 403

Reipurth, B., Bally, J., Fesen, R. A., \& Devine, D. 1998, Nature, 396, 343

Reipurth, B., Heathcote, S., Morse, J., Hartigan, P., \& Bally, J., 2002, AJ, 123, 362

Reipurth, B., Rodríguez, L.F., Anglada, G., \& Bally, J. 2004, AJ, in press

Shu, F. H., Najita, J. R., Shang, H., \& Li, Z.-Y. 2000, in Protostars and Planets $I V$, eds. V. Mannings, A. P. Boss, \& S. S. Russell (Tucson: University of Arizona Press), p.789

Solf, J. 1989, in ESO Conf. Proc. 33, Low-Mass Star Formation and Pre-Main Sequence Objects, ed. B. Reipurth (Garching: ESO), p.399

Solf, J. 1994, in ASP Conf. Ser. 57, Stellar and Circumstellar Astrophysics, ed.

G. Wallerstein \& A. Noriega-Crespo (San Francisco: ASP), p.22

Solf, J., \& Böhm, K.-H. 1999, ApJ, 523, 709 